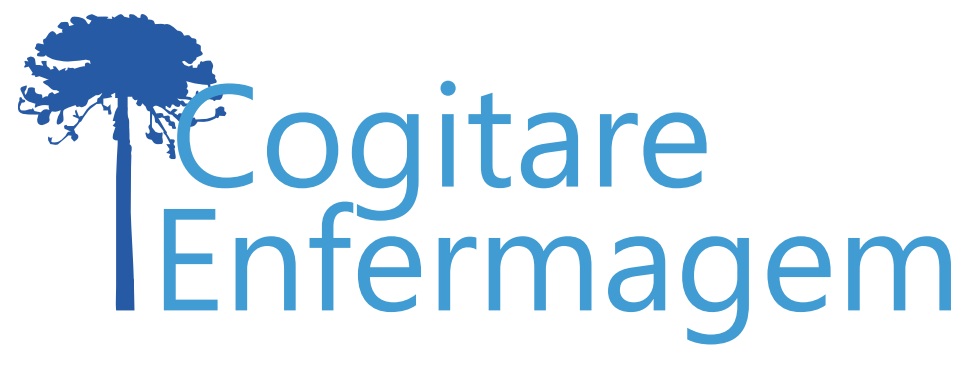

\title{
NOTIFICAÇÃO DA SÍFILIS ADQUIRIDA EM UMA SUPERINTENDÊNCIA REGIONAL DE SAÚDE DO SUL DE MINAS GERAIS*
}

Grazielle Miranda Freitas ${ }^{1}$, Murilo César do Nascimento², Edilaine Assunção Caetano de Loyola $^{3}$, André Silva Tavares ${ }^{4}$, Denismar Alves Nogueira ${ }^{5}$, Fábio de Souza Terra ${ }^{6}$

\section{RESUMO}

Objetivo: avaliar as notificações dos casos de sífilis adquirida em uma Superintendência Regional de Saúde do Sul de Minas Gerais.

Método: estudo transversal-descritivo-analítico dos 294 casos de sífilis notificados entre janeiro de 2010 e junho de 2017. Na coleta utilizou-se um instrumento elaborado pelos pesquisadores e os dados foram analisados com os testes Qui-quadrado de Pearson e Exato de Fisher. Estimou-se o odds ratio das variáveis, com o intervalo de confiança de $95 \%$.

Resultados: observou-se maior frequência de pessoas com faixa etária de 20 a 35 anos, 162 $(55,1 \%)$; sexo masculino, 203 (69\%); escolaridade de ensino médio completo, 58 (19,7\%); resultado do teste não-treponêmico reagente, 256 (87,1\%), 156 (53,1\%); classificação clínica em fase terciária, $89(30,3 \%)$. A variável sexo apresentou associação significativa com o comportamento sexual $(\mathrm{p}<0,001)$.

Conclusão: a avaliação das notificações foi alcançada, o que pode favorecer a detecção precoce, o diagnóstico apropriado e o tratamento imediato à pessoa.

DESCRITORES: Sistemas de Informação em Saúde; Notificação de Doenças; Sífilis; Monitoramento Epidemiológico; Enfermagem.

*Artigo extraído da dissertação de mestrado "Notificação da sífilis adquirida em uma Superintendência Regional de Saúde do sul de Minas Gerais". Universidade Federal de Alfenas, 2019.

\section{COMO REFERENCIAR ESTE ARTIGO:}

Freitas GM, Nascimento MC do, Loyola EAC de, Tavares AS, Nogueira DA,Terra F de S. Notificação da sífilis adquirida em uma superintendência regional de saúde do sul de Minas Gerais. Cogitare enferm. [Internet]. 2019 [acesso em "colocar data de acesso, dia, mês abreviado e ano"]; 24. Disponível em: http://dx.doi.org/10.5380/ ce.v24i0.62274.

Este obra está licenciado com uma Licença Creative Commons Atribuição 4.0 Internacional.

${ }^{1}$ Enfermeira. Mestranda em Enfermagem. Universidade Federal de Alfenas. Alfenas, MG, Brasil. 9

${ }^{2}$ Enfermeiro. Doutor em Enfermagem. Docente de Enfermagem da Universidade Federal de Alfenas. Alfenas, MG, Brasil.

${ }^{3}$ Enfermeira. Doutora em Ciências. Docente da Universidade José do Rosário Vellano. Alfenas, MG, Brasil. 1

${ }^{4}$ Geógrafo. Doutorando em Geociências Aplicadas. Universidade de Brasília. Brasília, DF, Brasil. ()

${ }^{5}$ Estatístico. Doutor em Estatística e Experimentação Agropecuária. Docente da Universidade Federal de Alfenas. Alfenas, MG, Brasil.

${ }^{6}$ Enfermeiro. Doutor em Enfermagem. Docente de Enfermagem da Universidade Federal de Alfenas. Alfenas, MG, Brasil. 


\title{
NOTIFICATION OF SYPHILIS ACQUIRED IN A REGIONAL HEALTH SUPERINTENDENCE OF SOUTH MINAS GERAIS
}

\begin{abstract}
Objective: To evaluate the notifications of syphilis cases acquired at a Regional Health Superintendence of Southern Minas Gerais.

Method: A descriptive-analytical cross-sectional study of the 294 syphilis cases reported between January 2010 and June 2017. In the collection an instrument elaborated by the researchers was used and the data were analyzed with the Pearson chi-square test and Fisher's exact test. Odds ratio variables were estimated with a confidence interval of $95 \%$.

Results: There was a higher frequency of people aged 20 to 35 years old, 162 (55.1\%); male, 203 (69\%); complete high school education, 58 (19.7\%); non-treponemal reagent test result, 256 (87.1\%), 156 (53.1\%); tertiary phase clinical classification, 89 (30.3\%). The gender variable was significantly associated with sexual behavior $(p<0.001)$.

Conclusion: The assessment of the notifications was achieved, which may favor early detection, appropriate diagnosis and prompt treatment to the person.
\end{abstract}

DESCRIPTORS: Health Information Systems; Disease Notification; Syphilis; Epidemiological Monitoring; Nursing.

\section{NOTIFICACIÓN DE SÍFILIS HECHA EN UNA SUPERINTENDENCIA REGIONAL DE SALUD DEL SUR DE MINAS GERAIS}

\begin{abstract}
RESUMEN
Objetivo: evaluar las notificaciones de los casos de sífilis adquirida en una Superintendencia Regional de Salud del Sur de Minas Gerais.

Método: estudio trasversal, descriptivo y analítico de los 294 casos de sífilis notificados entre enero de 2010 y junio de 2017. Se obtuvieron los datos por medio de un instrumento creado por los investigadores y se los analizaron por medio de los tests Chi cuadrado de Pearson y Exato de Fisher. Se estimó el odds ratio de las variables, con el intervalo de confianza de 95\%. Resultados: se observó mayor frecuencia de personas en la franja etaria de 20 a 35 años, 162 (55,1\%); sexo masculino, 203 (69\%); escolaridad de enseñanza secundaria completa, 58 $(19,7 \%)$; resultado del test no treponémico reagente, $256(87,1 \%), 156(53,1 \%)$; clasificación clínica en fase terciaria, $89(30,3 \%)$. La variable sexo presentó asociación significativa con el comportamiento sexual $(p<0,001)$.

Conclusión: se alcanzó la evaluación de las notificaciones, lo que sí puede favorecer la detección precoz, el diagnóstico apropiado y el tratamiento inmediato a la persona.
\end{abstract}

DESCRIPTORES: Sistemas de Información en Salud; Notificación de Enfermedades; Sífilis; Monitoreo Epidemiológico; Enfermería. 
É conhecido que existem fatores relevantes na transmissibilidade da sífilis relacionados a aspectos sociais, biológicos, culturais e comportamentais dos indivíduos. Tais fatores podem interferir na determinação da doença e na procura, por parte das pessoas acometidas, pelos serviços diagnósticos e de tratamento(1).

A sífilis persiste como problema de saúde pública, apesar de seu agente etiológico ter sido descoberto há séculos; da existência de métodos diagnósticos confiáveis e acessíveis, incluindo os testes rápidos; e da terapêutica disponível, com boa relação custoefetividade ${ }^{(2)}$.

Os últimos dados publicados pela Organização Mundial da Saúde (OMS) demonstram estimativa de ocorrência de 12 milhões de casos novos de sífilis por ano no mundo, sendo que entre 2016 e 2017 , houve um aumento de $31,8 \%$ no número de casos na população brasileira sexualmente ativa ${ }^{(3-4)}$.

O Ministério da Saúde (MS) recomenda que os profissionais de saúde, principalmente os médicos e os enfermeiros, estejam aptos a reconhecer as manifestações clínicas da sífilis, a interpretar os resultados dos exames laboratoriais e a monitorar a resposta ao tratamento, por meio dos serviços de vigilância. Mas nem sempre os profissionais estão preparados para abordar estas pessoas nos serviços de saúde ${ }^{(5)}$.

Quando os portadores e as reinfecções pela sífilis não são tratados adequadamente, o indivíduo pode ter como consequência o risco de resistência bacteriana. Assim, a próxima infecção poderá progredir para as formas mais graves, ou seja, as fases secundária e terciária. Ademais, existe o risco da evolução para a doença inflamatória pélvica, para as mulheres, e a infertilidade, para mulheres e homens ${ }^{(6)}$.

Destaca-se que no Brasil, nos últimos cinco anos, ocorreu aumento constante no número de casos de sífilis adquirida ${ }^{(7)}$. Compete aos gestores de políticas públicas de saúde fortalecer as ações para o controle da sífilis, com estratégias de rastreamento, diagnóstico e tratamento precoces, impedindo complicações, suavizando a morbidade, visando à melhor qualidade da saúde sexual e reprodutiva da população(8).

Assim, devido a serem incipientes os estudos sobre a sífilis adquirida e ser recente a inserção desse agravo como doença de notificação compulsória, o conhecimento do perfil epidemiológico de portadores de sífilis é importante para a população, para os profissionais da saúde, incluindo os enfermeiros, e para as políticas públicas de saúde. Isso devido ao fato de a detecção precoce possibilitar diagnóstico e tratamento imediatos, às pessoas acometidas e a seus parceiros, e orientação de práticas de prevenção que podem impedir a progressão da doença, quebrando a cadeia de transmissão.

Com isso, este estudo teve como objetivo avaliar as notificações dos casos de sífilis adquirida registradas em uma Superintendência Regional de Saúde do Sul (SRS) de Minas Gerais.

\section{MÉTODO}

Trata-se de um estudo transversal-descritivo-analítico, desenvolvido em uma SRS do Sul de Minas Gerais, que abrange 26 municípios, com população aproximada de 460 mil habitantes.

A população do estudo foi constituída de todos os casos de sífilis adquirida notificados na referida SRS, no período de janeiro de 2010 a junho de 2017, acompanhados da ficha de investigação, contabilizando 294 fichas. Esse período foi escolhido porque a notificação 
da sífilis adquirida iniciou no ano de 2010 e devido ao período de coleta de dados que ocorreu entre agosto a dezembro de 2017.

Para a coleta de dados foi utilizado um instrumento elaborado pelos pesquisadores, com base na ficha de investigação preconizada pela Secretaria Estadual de Saúde. Esse instrumento consta de três partes: dados sociodemográficos, com 10 variáveis; dados clínicos e laboratoriais, com 5 variáveis; e evolução do caso, com 2 variáveis.

A SRS solicitou a cada município que pertence à microrregião o envio, via e-mail, das fichas de investigação escaneadas, com os dados pessoais de cada paciente (nome e endereço) ocultados. Em posse dessas fichas, foram extraídas as informações e registradas no instrumento o qual, após o preenchimento completo, ficou em posse dos pesquisadores para posterior análise dos dados.

Os dados coletados foram agrupados em um banco de dados utilizando-se uma planilha eletrônica. Foi efetuada a dupla digitação para evitar possíveis erros de transcrição e, para análise estatística descritiva, foi utilizado o programa Statistical Package for the Social Science (SPSS), versão 20.0. Foram empregados os testes Qui-quadrado de Pearson e Exato de Fisher, com o nível de significância de $5 \%$. Após as análises, estimou-se o odds ratio das variáveis avaliadas, com intervalo de confiança de $95 \%$.

Este estudo foi aprovado pelo Comitê de Ética em Pesquisa por meio do Parecer número 2.197.263.

\section{RESULTADOS}

Constatou-se que no período de janeiro de 2010 a junho de 2017 foram enviadas 294 notificações com fichas de investigação de sífilis adquirida. $O$ ano de notificação mais frequente foi 2016, com 69 (23,5\%) fichas de investigação; e o ano com mais diagnósticos foi 2015 , com $66(22,4 \%)$ casos.

Evidenciou-se que a faixa etária mais ocorrente foi a de 20 a 35 anos, com 162 $(55,1 \%)$ fichas de notificação (média de 30,73 anos e mediana de 28 anos), seguida pela de 36 a 50 anos, com 54 (18,4\%) fichas. Com relação ao sexo, 203 (69\%) pessoas eram do sexo masculino. A raça/cor branca correspondeu a 157 (53,4\%) investigações, seguida pela parda, com $83(28,2 \%)$.

Quanto à escolaridade, os indivíduos notificados e investigados possuem ensino médio completo, totalizando $58(19,7 \%)$ das fichas de notificação, seguida pela $5^{\circ}$ a $8^{\circ}$ série incompleta do Ensino Fundamental, com $54(18,4 \%)$ das fichas. Referente à zona de moradia, $261(88,8 \%)$ dos registros indicaram a zona urbana; a ocupação do lar correspondeu a 34 $(11,6 \%)$ casos, seguidos de serviços gerais, com $30(10,2 \%)$ e estudantes com $28(9,5 \%)$ das fichas de notificação.

De acordo com as fichas de investigação analisadas, 39 (13,3\%) casos de sífilis tiveram antecedentes para este agravo. Desses casos, o tratamento foi realizado em $30(76,9 \%)$ pessoas, conforme apresentado na Tabela 1.

Tabela 1 - Distribuição das fichas de investigação da Superintendência Regional de Saúde, notificadas no período de janeiro de 2010 a junho de 2017, de acordo com as variáveis "antecedentes de sífilis" e "tratamento realizado". Alfenas, MG, Brasil, 2017 (continua) 


\begin{tabular}{lcc}
\hline Não preenchido & 11 & 3,7 \\
\hline Não & 210 & 71,4 \\
\hline Sim & 39 & 13,3 \\
\hline Ignorado $^{2}$ & 34 & 11,6 \\
\hline Tratamento realizado $^{+}$ & & \\
\hline Sim & 30 & 76,9 \\
\hline Não & 7 & 17,9 \\
\hline Ignorado $^{-}$ & 2 & 5,1
\end{tabular}

† Somente fichas que possuíam preenchimento do campo "sim" para variável antecedentes de sífilis ( $n=39)$.

Ao avaliar as fichas de investigação conforme a variável comportamento sexual, verificou-se que os casos de sífilis adquirida notificados e investigados eram de $120(40,8 \%)$ indivíduos (homens e mulheres) que mantêm relações sexuais com homens; $110(37,4 \%)$ pessoas (homens e mulheres) que praticam relaçõ̂es sexuais com mulheres; e $16(5,4 \%)$ casos (homens e mulheres) com histórico de relações tanto com homens quanto com mulheres.

Constatou-se que a variável sexo apresentou associação significativa com o comportamento sexual, ou seja, os casos de sífilis adquirida do sexo masculino apresentam mais chance de comportamento sexual para relações sexuais com homens e mulheres, conforme apresentado na Tabela 2.

Tabela 2 - Análise dos fatores associados ao comportamento sexual (relações sexuais com homens e relações sexuais com homens e mulheres), conforme a variável "sexo". Alfenas, MG, Brasil, 2017

\begin{tabular}{|c|c|c|c|c|c|}
\hline \multirow[t]{2}{*}{ Variável } & \multicolumn{2}{|c|}{ Comportamento sexual } & \multirow[t]{2}{*}{ Valor-p } & \multirow[t]{2}{*}{$\mathrm{OR}^{\dagger}$} & \multirow[t]{2}{*}{$I C \mp 95 \%$} \\
\hline & $\begin{array}{c}\text { Relações sexuais com } \\
\text { homens }\end{array}$ & $\begin{array}{c}\text { Relações sexuais com } \\
\text { homens e mulheres }\end{array}$ & & & \\
\hline \multicolumn{6}{|l|}{ Sexo } \\
\hline Masculino & $49(79,0 \%)$ & $13(21,0 \%)$ & $<0,001^{\S}$ & 1 & $0,007-0,431$ \\
\hline Feminino & $69(98,6 \%)$ & $1(1,4 \%)$ & & 0,055 & \\
\hline
\end{tabular}

Verificou-se que $256(87,1 \%)$ dos casos avaliados possuíam resultado do teste nãotreponêmico reagente. Dentre eles, constatou-se que os casos notificados apresentaram diferentes titulações. Quanto ao resultado do teste treponêmico, $156(53,1 \%)$ fichas de investigação possuíam resultado reagente, conforme Tabela 3. 
Tabela 3 - Distribuição das fichas de investigação da Superintendência Regional de Saúde, notificadas no período de janeiro de 2010 a junho de 2017, de acordo com as variáveis "resultado do teste nãotreponêmico", "titulações" e "resultado do teste treponêmico". Alfenas, MG, Brasil, 2017 (continua)

\begin{tabular}{lcc} 
Variáveis & $\mathbf{n}$ & $\%$ \\
\hline Resultado do Teste não-treponêmico & & \\
\hline Não preenchido & 18 & 6,1 \\
\hline Reagente & 256 & 87,1 \\
\hline Não Reagente & 5 & 1,7 \\
\hline Não realizado & 14 & 4,8 \\
\hline Ignorado & 1 & 0,3 \\
\hline Titulações ${ }^{\dagger}$ & & \\
\hline Não preenchido & 21 & 8,2 \\
\hline Titulação 1 ao 8 & 87 & 34 \\
\hline Titulação 16 & 44 & 17,2 \\
\hline Titulação 32/36 & 46 & 18 \\
\hline Titulação 64 & 42 & 14,3 \\
\hline Titulação 128 ou mais & 16 & 6,3 \\
\hline Resultado do Teste treponêmico & & \\
\hline Não preenchido & 36 & 12,2 \\
\hline Reagente & 156 & 53,1 \\
\hline Não Reagente & 13 & 4,4 \\
\hline Não realizado & 70 & 23,8 \\
\hline lgnorado & 19 & 6,5 \\
\hline
\end{tabular}

† Somente fichas que possuíam preenchimento do campo reagente para a variável Resultado do teste não-treponêmico ( $n=256$ ).

Ao verificar a distribuição dos casos de sífilis adquirida conforme a variável classificação clínica, constatou-se que 89 (30,3\%) indivíduos possuíam doença em sua fase terciária. O esquema de tratamento realizado mais frequente foi o de Penicilina G benzatina 7.200.000 UI, correspondendo a $111(37,8 \%)$ casos. Quanto à classificação final do caso, 233 (79,3\%) foram casos confirmados de portadores de sífilis adquirida, conforme evidenciado na Tabela 4.

Tabela 4 - Distribuição das fichas de investigação da Superintendência Regional de Saúde, notificadas no período de janeiro de 2010 a junho de 2017, de acordo com as variáveis "classificação clínica", "esquema de tratamento realizado" e "classificação final do caso". Alfenas, MG, Brasil, 2017 (continua)

\begin{tabular}{lll} 
Variáveis & $\mathbf{n}$ & $\%$ \\
\hline Classificação clínica & & \\
\hline Não preenchido & 43 & 14,6 \\
\hline Primária & 76 & 25,9 \\
\hline Secundária & 36 & 12,2 \\
\hline
\end{tabular}




\begin{tabular}{lcc}
\hline Terciária & 89 & 30,3 \\
\hline Latente & 22 & 7,5 \\
\hline Ignorado & 28 & 9,5 \\
\hline Esquema de Tratamento realizado & & \\
\hline Não preenchido & 27 & 9,2 \\
\hline Penicilina G benzatina 2.400.000 UI & 87 & 29,6 \\
\hline Penicilina G benzatina 4.800.000 UI & 18 & 6,1 \\
\hline Penicilina G benzatina 7.200.000 UI & 111 & 37,8 \\
\hline Outro esquema & 10 & 3,4 \\
\hline Não realizado & 25 & 8,5 \\
\hline lgnorado & 16 & 5,4 \\
\hline Classificação final do caso & & \\
\hline Não preenchido & 51 & 17,3 \\
\hline Confirmado & 233 & 79,3 \\
\hline Descartado & 10 & 3,4
\end{tabular}

As variáveis faixa etária, escolaridade e zona de moradia não apresentaram associação significativa com o comportamento sexual (respectivamente, $p=0,749, p=0,528, p=0,608$ ). As variáveis faixa etária, sexo, escolaridade e zona de moradia também não apresentaram associação significativa com antecedentes de sífilis (respectivamente, $p=0,377, p=0,152$, $p=0,143, p=0143$ ) e com classificação clínica (respectivamente, $p=0,970, p=0,622, p=0,066$, $p=1,000)$. Não houve ainda associação significativa entre a variável antecedentes da sífilis com as variáveis comportamento sexual $(p=1,000)$ e a variável comportamento sexual com a variável classificação clínica $(p=0,821)$.

\section{DISCUSSÃO}

Neste estudo, a amostra foi composta por casos na faixa etária de 20 a 35 anos, no sexo masculino, na raça/cor branca, com escolaridade em ensino médio completo, tendo a zona urbana como moradia e a ocupação do lar. Esses achados assemelham-se aos resultados de outros estudos ${ }^{(9-12)}$, apresentados a seguir.

Em uma investigação realizada na Alemanha, foi observado que $94 \%$ dos casos notificados eram do sexo masculino(9). Em pesquisa realizada em São Paulo houve predominância do sexo masculino, 97,8\%. Com isso, percebe-se que os homens são mais acometidos pela sífilis em comparação às mulheres, mesmo em outros países.

Nesse mesmo estudo, houve maior frequência da faixa etária de 25 a 34 anos, 40,3\%; da escolaridade ensino médio completo, $33,4 \%$, e da raça cor branca, com $59,9 \%$ dos casos avaliados $^{(10)}$. Num inquérito realizado na Bahia com 33.665 pessoas, foi observado, em $100 \%$ dos casos de sífilis adquirida em adolescentes, que estes residiam na zona urbana ${ }^{(11)}$.

Em um estudo realizado em Nova lorque, no ano de 2015, com uma população de 2805 homens portadores de sífilis, constatou-se que $4 \%$ dos homens tinham mais de um diagnóstico de sífilis, e observou-se que $67,2 \%$ dos homens faziam sexo com homens e sua menor proporção era de $13,3 \%$ de homens que faziam sexo com mulheres ${ }^{(12)}$. Na presente investigação, $13,3 \%$ dos casos avaliados apresentaram antecedentes de sífilis, e 79,9\% dos 
homens mantêm relações sexuais com homens.

Com relação às características de comportamento sexual, estudo encontrou um percentual de $88,1 \%$ de homens que fazem sexo com homens ${ }^{(10)}$, valores acima do encontrado na presente pesquisa.

Devido aos padrões de gênero hegemônicos da sociedade, o sexo masculino fica mais exposto aos riscos de contrair Infecção Sexualmente Transmissível (IST), uma vez que este tem um comportamento sexual de maior susceptibilidade associado à iniciação da vida sexual precoce, à exposição a vários parceiros sexuais, à homossexualidade e ao nãouso do preservativo ${ }^{(13)}$.

Dessa maneira, vale refletir sobre os determinantes sociais em saúde que mencionam um conjunto de informações elencadas em categorias de fatores comportamentais, biológicos e circunstanciais. Destaca-se como exemplo desses fatores as condições de vida, de acesso a ensino de qualidade, do núcleo familiar, das atitudes comportamentais na vida sexual, entre outros, além da existência dos fatores psicossociais ${ }^{(14)}$.

De acordo com a análise das associações das variáveis, constatou-se que o sexo masculino apresentou associação significativa com o comportamento sexual. Assim, faz-se importante discutir alguns aspectos que podem estar ligados a essa associação de variáveis em destaque.

Dados encontrados no inquérito desenvolvido no Canadá levaram à constatação de que o aumento de sífilis adquirida no homem pode estar associado com o comportamento sexual, em que, cada vez mais, há acréscimo de homens que fazem sexo com homens, uma vez que essas causas neste grupo específico podem ser multifatoriais e mais complexas do que em outros grupos ${ }^{(15)}$.

Esses achados se manifestaram em estudo realizado com adolescentes na Bahia, uma vez que a multiplicidade de parceiros sexuais foi de maior frequência entre indivíduos do sexo masculino, aumentando assim o risco para o contágio com outras IST, além da sífilis adquirida ${ }^{(11)}$.

Com o passar dos anos, o comportamento sexual dos homens vem contribuindo substancialmente no crescente número de casos de sífilis adquirida, verificado em todas as idades, mas com destaque entre os adultos jovens ${ }^{(16)}$. Vale acrescentar que os homens tendem a procurar menos pelos serviços de saúde mediante as dificuldades por eles encontradas, como o horário de funcionamento dos serviços, uma vez que muitas vezes não possuem disponibilidade em sua vida cotidiana de trabalho para agendar os atendimentos ou até mesmo para esperar a consulta que pode ser demorada ${ }^{(17)}$.

Nesse contexto, o enfermeiro tem papel de incentivar hábitos comportamentais seguros na intenção de quebrar a cadeia de transmissão, bem como criar estratégias de prevenção, educação e aconselhamento, a fim de informar às pessoas sobre os riscos de aquisição dessas infecções e suas possíveis complicações ${ }^{(18)}$.

Com relação às variáveis clínicas e laboratoriais, foram constatados no presente estudo casos com resultados do teste não-treponêmico reagente, com titulação de 1 a 32, classificação clínica da doença em fase terciária, com esquema de tratamento realizado com Penicilina G benzatina 7.200.000 Unidades Internacionais (UI) e classificação final como confirmados portadores de sífilis adquirida.

Em investigação realizada em São Paulo, dos casos notificados de sífilis adquirida, $90,6 \%$ apresentaram resultados do teste treponêmino reagente com titulação de $1 / 32^{(10)}$, corroborando com o presente estudo.

A classificação clínica mais frequente foi a terciária. Pesquisa realizada em Nova lorque com registros de Vigilância dos agravos em estudo constatou que somente $14 \%$ dos homens eram portadores de sífilis em sua fase terciária(12), o que corrobora com os 
achados da investigação desenvolvida em Londres, com homens diagnosticados com sífilis em suas fases iniciais (primária e secundária) ${ }^{(19)}$.

Destaque-se que o ápice da transmissão da sífilis acontece quando a infecção está em sua fase primária e secundária, sendo assim, o rastreamento bem realizado pelos profissionais de saúde, sobretudo os enfermeiros, e o tratamento com acompanhamento dos resultados são primordiais para inibir essa transmissão(20).

Em relação à variável esquema de tratamento, em pesquisa internacional verificaramse indivíduos que foram tratados com 2,4 milhões Ul de penicilina benzatina por via intramuscular, uma vez que foram diagnosticados em fase primária e secundária da sífilis ${ }^{(20)}$. Esses achados foram discordantes com os resultados do presente estudo, mas justifica-se pelo fato de predominar pessoas diagnosticadas com esse agravo na fase terciária.

O MS preconiza para o tratamento da sífilis a utilização da penicilina como o medicamento de escolha. Na sífilis primária, na secundária e na latente recente, devendo esta ter até um ano de duração, o tratamento deve ser realizado com a penicilina $G$ benzatina, 2,4 milhões UI, via intramuscular (IM) e em dose única (1,2 milhão Ul em cada glúteo ${ }^{(5)}$. No que tange à sífilis latente tardia com mais de um ano de duração ou latente com duração ignorada e na sífilis terciária deve ser utilizada a penicilina $G$ benzatina, 2,4 milhões UI, IM, sendo 1,2 milhão UI em cada glúteo, semanalmente e por três semanas ${ }^{(5)}$.

Entretanto, autores trazem que outros tratamentos podem ser tão eficazes quanto a penicilina para tratamento da sífilis, principalmente se esta estiver em sua fase primária. Contudo, os pacientes devem receber acompanhamento rigoroso, uma vez que, se houver falha nesse processo, o agravo pode ser identificado prematuramente ${ }^{(21)}$.

Destaca-se a grande dificuldade de encontrar nos estudos sobre sífilis adquirida a abordagem da variável classificação final do caso. Com isso, a comparação desta variável ocorreu com pesquisas desenvolvidas com a sífilis congênita, em que quase a totalidade das pessoas avaliadas apresentaram o caso confirmado ${ }^{(22-23)}$.

Asífilis adquirida se apresenta como uma doença silenciosa, muitas vezes assintomática, contribuindo para que os casos continuem difíceis de serem diagnosticados, tratados e, consequentemente, notificados/investigados de forma apropriada. Sendo assim, se não ofertado o tratamento adequado e o diagnóstico precoce, essa doença pode trazer um impacto negativo na vida do paciente. Ademais, pode trazer consequências sociais aos portadores, levando a impactos financeiros com o uso de medicamentos, afastamento das funções trabalhistas, preconceito da sociedade, entre outros efeitos que o adoecimento pode trazer ${ }^{(24)}$.

Com isso, se não tratada de forma adequada, a sífilis adquirida pode evoluir para as fases subsequentes da doença, fazendo com que o comprometimento da saúde do indivíduo portador seja cada vez maior. Desse modo, a detecção precoce e o tratamento imediato da sífilis são essenciais na prevenção de novas transmissões e controle da doença, assim como no impedimento de complicações severas propiciando esclarecimentos ao portador, principalmente nos aspectos ligados a mudanças de comportamento sexual e na exposição de risco ${ }^{(24)}$.

Neste contexto, vale discutir a atuação do enfermeiro na atividade do aconselhamento, visto que esta é uma etapa primordial no processo de testagem, pelo qual este profissional é o maior responsável. Há necessidade do enfermeiro ofertar suporte emocional adequado no pós-teste, e na implantação de capacitações e atividades de educação permanente voltadas para esse tema, além do envolvimento dos demais membros da equipe ${ }^{(25)}$.

Com isso, faz-se necessário que todos os profissionais de saúde envolvidos façam aconselhamentos abrangentes, valendo-se de histórias sexuais atendidas anteriormente, e tenham a atitude de rastrear as IST na população(26).

Ainda, ressalta-se que o enfermeiro desempenha inúmeras atividades relevantes 
para a prevenção das IST. Dessa forma, os portadores podem ser encaminhados por este profissional, de maneira mais rápida e efetiva, aos outros níveis e serviços de atenção à saúde ${ }^{(27)}$.

O presente estudo apresentou a limitação de que foram avaliadas apenas as fichas de investigação de sífilis adquirida enviadas na íntegra para a SRS, uma vez que no Sistema de Informação de Agravos de Notificação a ficha não está disponibilizada em sua integralidade, existindo somente como ficha de conclusão de casos. Diante disso, afirmase que há necessidade urgente da inserção da ficha de investigação da sífilis adquirida original nesse sistema, uma vez que, sem os dados competentes no sistema, o MS pode ficar impossibilitado de conhecer os dados clínicos e laboratoriais dessa população no Estado de Minas Gerais.

\section{CONCLUSÃO}

Conclui-se que a sífilis adquirida ocorreu mais em homens, jovens, e diagnosticada na fase terciária. Os homens apesentaram mais chance de comportamento sexual para relações com homens e relações com homens e mulheres, tendo oportunidade mais abrangente de adquirir esse agravo ou qualquer outra IST.

Diante disso, este estudo contribui para o real conhecimento do perfil epidemiológico da sífilis adquirida, colaborando para a construção de informações que favoreçam o desenvolvimento de ações conjuntas entre os serviços de atenção à saúde e os gestores de cada localidade, a fim de ofertar o diagnóstico e o tratamento precoces para inibir a transmissão.

\section{REFERÊNCIAS}

1. Shah BJ, Karia DR, Pawara CL. Syphilis: Is it making resurgence? Indian J Sex Transm Dis AIDS [Internet]. 2015 [acesso em 05 ago 2018]; 36(2). Disponível em: http://dx.doi.org/10.4103/02537184.167170.

2. Cunha ARC da, Merchan-Hamann E. Sífilis em parturientes no Brasil: prevalência e fatores associados, 2010 a 2011. Rev Panam Salud Publica. [Internet]. 2015 [acesso em 10 ago 2018]; 38(6). Disponível em: https://scielosp.org/pdf/rpsp/2015.v38n6/479-486/pt .

3. World Health Organization (WHO). Progress report, 2010. [Internet]. Geneva: WHO; 2010 [acesso em 25 ago 2018]. Disponível em: https://www.who.int/reproductivehealth/topics/rtis/GlobalData cs pregnancy.pdf .

4. Ministério da Saúde (BR). Secretaria de Vigilância em Saúde. Departamento de DST, Aids e Hepatites Virais. Boletim Epidemiológico Sífilis. [Internet]. Brasília: Ministério da Saúde; 2018 [acesso em 01 set 2019]. Disponível em: http://www.aids.gov.br/pt-br/pub/2018/boletim-epidemiologico-de-sifilis-2018.

5. Ministério da Saúde (BR). Secretaria de Vigilância em Saúde. Departamento de DST, Aids e Hepatites Virais. Protocolo clínico e diretrizes terapêuticas para prevenção da transmissão vertical de HIV, Sífilis e Hepatites Virais. [Internet]. Brasília: Ministério da Saúde; 2015. Modificado em junho de 2019 [acesso em 30 jul 2019]. Disponível em: http://www.aids.gov.br/pt-br/pub/2015/protocolo-clinico-e-diretrizesterapeuticas-para-atencao-integral-pessoas-com-infeccoes.

6. Ministério da Saúde (BR). Secretaria de Vigilância em Saúde. Departamento de DST, Aids e Hepatites Virais. Manual Técnico para Diagnóstico da Sífilis. [Internet]. Brasília: Ministério da Saúde; 2016 [acesso em 30 jul 2019]. Disponível em: http://www.aids.gov.br/pt-br/pub/2016/manual-tecnico-para-diagnostico-dasifilis. 
7. Ministério da Saúde (BR). Secretaria de Vigilância em Saúde. Departamento de DST, Aids e Hepatites Virais. Boletim Epidemiológico Sífilis. [Internet]. Brasília: Ministério da Saúde; 2017 [acesso em 24 ago 2018]. Disponível em: http://www.aids.gov.br/pt-br/pub/2017/boletim-epidemiologico-de-sifilis-2017.

8. Pinto VM, Tancredi MV, Alencar HDR de, Camolesi E, Holcman MM, Grecco JP, et al. Prevalence of Syphilis and associated factors in homeless people of Sao Paulo, Brazil, using a Rapid Test. Rev. bras. epidemiol. [Internet]. 2014 [acesso em 10 ago 2018]; 17(2). Disponível em: http://dx.doi. org/10.1590/1809-4503201400020005ENG .

9. Bremer $V$, Marcus U, Hamouda O. Syphilis on the rise again in Germany - results from surveillance data for 2011. Eurosurveillance [Internet]. 2012 [acesso em 15 ago 2018]; 17(29). Disponível em: https://www. eurosurveillance.org/content/10.2807/ese.17.29.20222-en .

10. Luppi CG, Gomes SEC, Silva RJC da, Ueno AM, Santos AMK dos, Tayra A, et al. Factors associated with HIV co-infection in cases of acquired syphilis reported in a Reference Center for Sexually Transmitted Diseases and AIDS in the municipality of São Paulo, Brazil, 2014. Epidemiol. Serv. Saude. [Internet]. 2018 [acesso em 08 ago 2018]; 27(1). Disponível em: http://dx.doi.org/10.5123/s1679-49742018000100008.

11. Monteiro MOP, Costa MCO, Vieira GO, Silva AL. Fatores associados à ocorrência de sífilis em adolescentes do sexo masculino, feminino e gestantes de um Centro de Referência Municipal/CRM DST/HIV/ AIDS de Feira de Santana, Bahia. Adolesc. Saude. [Internet]. 2015 [acesso em 19 ago 2018]; 12(3). Disponível em: http://www.adolescenciaesaude.com/detalhe artigo.asp?id=520 .

12. Pathela P, Braunstein SL, Blank S, Shepard C, Schillinger JA. The High Risk of an HIV Diagnosis Following a Diagnosis of Syphilis: A Population-level Analysis of New York City Men. CID. [Internet]. 2015 [acesso em 22 ago 2018]; 61(2). Disponível em: https://doi.org/10.1093/cid/civ289.

13. Taquette ST, Rodrigues $A$ de O, Bortolotti LR. HIV infection in male adolescents: a qualitative study. Cien Saude Colet [Internet]. 2015 [acesso em 22 ago 2018]; 20(7). Disponível em: http://dx.doi. org/10.1590/1413-81232015207.18102014.

14. Borde E, Hernandez-Alvarez M, Porto MF de S. Uma análise crítica da abordagem dos Determinantes Sociais da Saúde a partir da medicina social e saúde coletiva latino-americana. Saude debate. [Internet]. 2015 [acesso em 24 ago 2018]; 39(106). Disponível em: http://dx.doi.org/10.1590/01031104201510600030023.

15. Choudhri, Y, Miller J, Sandhu J, Leon A, Aho J. Infectious and congenital syphilis in Canada, 2010-2015. Surveillance. [Internet]. 2018 [acesso em 21 set 2018]; 44(2). Disponível em: https://doi. org/10.14745/ccdr.v44i02a02.

16. Patton ME, Su JR, Nelson R, Weinstock H. Primary and Secondary Syphilis — United States, 20052013. MMWR. [Internet]. 2014 [acesso em 25 set 2018]; 63(18). Disponível em: https://www.cdc.gov/ mmwr/preview/mmwrhtml/mm6318a4.htm.

17. Sousa DHAV, Lima MAS, Vieira KFL, Saldanha AAW. Os homens e as práticas de cuidado em saúde. Gênero e Direito. [Internet]. 2015 [acesso em 25 set 2018]; 4(1). Disponível em: http://dx.doi. org/10.18351/2179-7137/ged.2015n1p397-415.

18. Ferraz LM, Martins ACS. Atuação do enfermeiro no diagnóstico e no tratamento do herpes genital, na Atenção Primária à Saúde. Rev. APS. [Internet]. 2014 [acesso em 31 ago 2018]; 17(2). Disponível em: https://aps.ufjf.emnuvens.com.br/aps/article/view/2006/797.

19. Girometti N, Gutierrez A, Nwokolo N, McOwan A, Whitlock G. High HIV incidence in men who have sex with men following an early syphilis diagnosis: is there room for pre-exposure prophylaxis as a prevention strategy?. Sex Transm Infect. [Internet]. 2016 [acesso em 09 set 2018]; 93(Suppl 1). Disponível em: http://dx.doi.org/10.1136/sextrans-2016-052865.

20. Abara, WE, Hess KL, Fanfair RN, Bernstein KT, Paz-Bailey G. Syphilis Trends among Men Who Have Sex with Men in the United States and Western Europe: A Systematic Review of Trend Studies Published between 2004 and 2015. PLoS ONE. [Internet]. 2016 [acesso em 05 set 2018]; 11(7). Disponível em: https://doi.org/10.1371/journal.pone.0159309. 
21. Liu H, Han Y, Chen X, Bai L, Guo S, Li L, et al. Comparison of efficacy of treatments for early syphilis: A systematic review and network meta-analysis of randomized controlled trials and observational studies. PLoS ONE. [Internet]. 2017 [acesso em 11 set 2018]; 12(6). Disponível em: https://doi.org/10.1371/ journal.pone.0180001.

22. Teixeira LO, Belarmino V, Gonçalves V, Mendoza-Sassi A. Tendência temporal e distribuição espacial da sífilis congênita no estado do Rio Grande do Sul entre 2001 e 2012. Cien Saude Colet. [Internet]. 2018 [acesso em 15 set 2018]; 23(8). Disponível em: http://dx.doi.org/10.1590/1413-81232018238.25422016.

23. Signor M, Spagnolo LML, Tomberg JO, Gobatto M, Stofel NS. Distribuição espacial e caracterização de casos de sífilis congênita. Rev. enferm. UFPE on line. [Internet]. 2018 [acesso em 18 set 2018]; 12(2). Disponível em: https://periodicos.ufpe.br/revistas/revistaenfermagem/article/view/230522/27844.

24. Vaulet LG, Morando N, Casco R, Melgar A, Nacher S, Fermepin MR, et al. Evaluation of the utility of a rapid test for syphilis at a sexually transmitted disease clinic in Buenos Aires, Argentina. Rci. Rep. [Internet]. 2018 [acesso em 31 jul 2019]; 8(5). Disponível em: https://www.ncbi.nlm.nih.gov/pmc/articles/ PMC5954144/pdf/41598 2018 Article 25941.pdf.

25. Araújo, WJ, Quirino EMB, Pinho CM, Andrade MS. Perception of nurses who perform rapid tests in Health Centers. Rev. bras. enferm. [Internet]. 2018 [acesso em 01 set 2018]; 71(Suppl1). Disponível em: http://dx.doi.org/10.1590/0034-7167-2017-0298.

26. Ferreira C de O, Viana ASA, Silva AA da, Rezende REA de, Gomes AVT de M, Davoglio RS. Perfil epidemiológico dos usuários de um Centro de Testagem e Aconselhamento da Bahia. Rev. baiana saúde pública. [Internet]. 2016 [acesso em 21 set 2018]; 40(2). Disponível em: https://doi.org/10.22278/23182660.2016.v40.n2.a1980.

27. Oliveira JAS de, Afonso TMO. Teste Rápido para o Diagnóstico de HIV na Atenção Primária à Saúde e a importância da atuação do Enfermeiro. UNIT Interfaces Científicas. [Internet]. 2017 [acesso em 02 set 2018]; 1(1). Disponível em: https://eventos.set.edu.br/index.php/cie/article/view/5714/2343.

Recebido: 09/10/2018

Finalizado: 11/09/2019

Autor Correspondente:

Grazielle Miranda Freitas

Universidade Federal de Alfenas

R. Gabriel Monteiro da Silva, 700 - 37130-001 - Alfenas, MG, Brasil

E-mail: grazimiranda85@gmail.com

\section{Contribuição dos autores:}

Contribuições substanciais para a concepção ou desenho do estudo; ou a aquisição, análise ou interpretação de dados do estudo - GMF, MCN, EACL, AST, DAN, FST

Elaboração e revisão crítica do conteúdo intelectual do estudo - GMF, MCN, EACL, FST

Aprovação da versão final do estudo a ser publicado - GMF, MCN, EACL, AST, DAN, FST

Responsável por todos os aspectos do estudo, assegurando as questões de precisão ou integridade de qualquer parte do estudo - GMF, FST 